\title{
Balkanologie
}

Balkanologie Revue d'études pluridisciplinaires

Vol. XI, $n^{\circ}$ 1-2 | 2008

Volume XI Numéro 1-2

\section{Les mobilités internes en Bulgarie, 1989-2005}

\section{Rossitza Guentcheva}

Traducteur : Nadège Ragaru et Sandrine Bochew

\section{(2) OpenEdition}

\section{Journals}

Édition électronique

URL : http://journals.openedition.org/balkanologie/1473

DOI : 10.4000/balkanologie.1473

ISSN : 1965-0582

Éditeur

Association française d'études sur les Balkans (Afebalk)

Référence électronique

Rossitza Guentcheva, «Les mobilités internes en Bulgarie, 1989-2005 », Balkanologie [En ligne], Vol. XI, n 1-2 | 2008, mis en ligne le 31 décembre 2008, consulté le 17 décembre 2020. URL : http:// journals.openedition.org/balkanologie/1473 ; DOI : https://doi.org/10.4000/balkanologie.1473

Ce document a été généré automatiquement le 17 décembre 2020.

(c) Tous droits réservés 


\title{
Les mobilités internes en Bulgarie, 1989-2005
}

\author{
Rossitza Guentcheva
}

Traduction : Nadège Ragaru et Sandrine Bochew

\section{NOTE DE L'ÉDITEUR}

Traduit du bulgare par Nadège Ragaru et Sandrine Bochew

1 Le présent article vise à dessiner les tendances des migrations internes en Bulgarie au cours de la période 1989-2005 tout en les comparant à celles de l'avant-1989. Les données statistiques utilisées sont issues des deux derniers recensements, des annuaires de l'Institut national statistique (NSI) et des recherches scientifiques consacrées à cette thématique en Bulgarie. Les flux migratoires seront analysés par âge, par sexe, par niveau d'éducation, par appartenance ethnique et en fonction de l'occupation économique des migrants; l'étude fera également apparaître des changements intervenus dans les directions des mobilités (ville-ville, ville-village, village-ville et village-village). Une attention plus spécifique sera accordée aux migrations entre départements et au sein des départements, tout comme aux déplacements de travail quotidiens. Nous discuterons également les politiques publiques mises en œuvre par l'Etat afin d'encourager les circulations internes, à l'image des programmes de renforcement de la mobilité de la main d'œuvre. Enfin, il nous semble indispensable de lier l'étude des mobilités internes à l'analyse des migrations internationales, même si une telle démarche se heurte à des difficultés sur lesquelles nous reviendrons.

2 Ces dix-huit dernières années, le problème des migrations internes en Bulgarie a rarement retenu l'attention des chercheurs. À la différence de leurs collègues de la période précédente, sociologues, anthropologues, économistes et politistes bulgares ont avant tout cherché à décrire et à interpréter les processus d'émigration. Avant le changement de régime, les migrations externes des citoyens bulgares n'étaient pas 
seulement strictement surveillées, contrôlées et régulées par les institutions de l'État socialiste $^{1}$; leur champ et leur intensité étaient également limités pour des raisons politiques et économiques. L'étude des mouvements de population vers l'étranger constituait un thème interdit aux chercheurs en sciences sociales. En outre, par leur ampleur, par leur intensité, les migrations internes revêtaient un caractère nettement plus visible et exerçaient un effet incomparable sur le développement de la société. Jusqu'en 1989, le contexte politique et la nature même des migrations dominantes ont ainsi tous deux orienté la recherche vers l'étude des mouvements de population intervenant à l'intérieur de l'État. Ce, au détriment d'une exploration des migrations externes. Les processus d'industrialisation et d'urbanisation, leurs effets sur les structures familiales, la distribution territoriale de la main d'œuvre, la croissance naturelle de la population, ainsi que sa mobilité sociale, ont alors figuré parmi les principales thématiques développées.

3 Après 1989, la suppression des restrictions aux sorties de territoire ${ }^{2}$ a entraîné un renouvellement du champ des études. Le regard des chercheurs s'est, comme celui de l'opinion publique, déplacé vers des migrations internationales particulièrement intenses fin 1989 et au début des années 1990. Le boom migratoire de la décennie 1990, provoqué aussi bien par la nouvelle liberté de circulation que par les difficultés associées à la transition politique et économique, s'est imposé durablement dans les quotidiens, les media électroniques et l'espace public. Jusqu'à ce jour, les départs des migrants continuent à servir d'explication à la fuite des cerveaux, à la crise démographique, à la baisse de la population en âge de travailler et au vieillissement de la nation, tandis que la solution est souvent recherchée dans un encouragement au retour des migrants (depuis l'Espagne, l'Italie, etc.) ou dans des programmes d'aide à l'installation en Bulgarie des Bulgares ethniques vivant en dehors du pays (Ukraine, Moldavie, etc. ${ }^{3}$ ). Dans ce contexte, les mobilités internes sont devenues un thème d'étude marginal après 1989 et rares sont les discussions publiques consacrées à leurs principales tendances, à leurs causes comme à leurs effets sociaux. Assurément, statisticiens et démographes continuent à étudier la mobilité territoriale interne et l'Institut national statistique a organisé la collecte de données relatives aux mouvements internes de population lors des recensements de 1992 et de 2001, ainsi qu'en dehors d'eux. Toutefois, dans les analyses des statisticiens et des démographes, les problèmes de mobilité interne sont principalement envisagés dans leurs aspects démographiques ${ }^{4}$. À quelques exceptions près, ces dernières ne sont pas appréhendées en tant qu'enjeu économique et social.

4 Si l'intérêt pour les migrations dans les débats publics et universitaires bulgares peut aisément s'expliquer au regard des processus politiques, économiques et sociaux intervenus dans le pays, on rappellera que cette tendance n'est pas propre à la Bulgarie. En Europe et aux États-Unis, ces vingt dernières années, maints sociologues, anthropologues, politistes, historiens et économistes se sont consacrés aux émigrations et immigrations, aux migrations transfrontalières et transcontinentales, ainsi qu'à la constitution de champs sociaux transnationaux. Les processus de globalisation et l'intensification sans précédent des circulations - qui a été vue, jusque récemment, comme sa résultante - ont promu des enjeux tels que l'intégration et l'assimilation des migrants, les liens sociaux multiples qu'ils entretiennent avec le pays d'accueil comme avec leur État d'origine, le rôle économique des transferts d'argent, etc. Le mouvement transfrontalier des gens, biens, capitaux et idées se situe ainsi au cœur des concepts de "flux culturels", "transnationalisme", "hybridité", "zones de contacts" et 
"communautés diasporiques" 5 . À la différence des migrations impliquant un franchissement des frontières étatiques, dans cette littérature, les circulations se déployant à l'intérieur des territoires nationaux reçoivent une attention modeste.

\section{Les mobilités internes en Bulgarie entre 1944 et 1989 : exode rural et urbanisation}

Bien que, dans les États socialistes, la mobilité interne ait été tenue pour la seule forme de circulation envisageable en l'absence de liberté de mouvement à l'international, cette migration n'était ni libre, ni illimitée, pas davantage indépendante. Les déplacements des citoyens bulgares à l'intérieur du territoire étaient - tout comme les migrations externes - très régulés, contrôlés et surveillés, quoiqu'avec des instruments et selon des mécanismes différents ${ }^{6}$. L'introduction de restrictions à l'installation dans la capitale, Sofia, date d'avant la fin de la seconde Guerre mondiale. Un régime de domiciliation (mestožiteltsvo) a été institué peu après. Progressivement, mais irrésistiblement au cours des années 1950-1960, dans un nombre croissant de municipalités l'arrivée de nouveaux habitants a été soumise à des conditions sociales et professionnelles, de sorte qu'au milieu des années 1970 l'obtention d'une autorisation de domiciliation constituait un préalable à toute installation en ville (à l'exception de quelque 30 bourgades de taille modeste ou situées en zone frontalière). Des mesures administratives, telles que le retrait d'autorisation de domiciliation permanente, la suspension d'une domiciliation temporaire ou encore la relégation forcée à l'intérieur du pays, ont permis à l'État d'intervenir directement sur les mobilités internes.

6 Parallèlement au renforcement de cet arsenal administratif, le pouvoir socialiste a mis en oeuvre une politique d'industrialisation, d'urbanisation et de collectivisation de l'agriculture à grande échelle, qui est entrée en conflit avec sa gestion des domiciliations dans les grandes et petites communes. Les mesures restrictives adoptées afin de réglementer l'accès aux biens publics (rares et précieux) proposés en milieu urbain (les écoles gratuites, les crèches, les soins médicaux, les logements à prix subventionnés - au nom du principe selon lequel tout citoyen socialiste y a également droit -, etc.), entrent en effet en contradiction avec les besoins en main d'œuvre des chantiers de construction de l'industrie et du bâtiment qui se multiplient dans la capitale et les villes bulgares en pleine expansion? ${ }^{7}$. Au moment même où se précise et s'élargit le contrôle administratif sur les domiciliations en ville, la Bulgarie connaît une urbanisation rapide associée à d'intenses mouvements de population. Ces processus sont particulièrement dynamiques entre le milieu des années 1950 et des années 1960. Le point culminant est atteint entre les recensements de 1956 et de 1965, avec près d'un million et demi de changements de lieux de résidence permanente ${ }^{8}$. Au cours des dix années suivantes, 1,304 millions de personnes adopteront une nouvelle domiciliation. Pendant la période 1976-1985, les migrations internes perdent en ampleur et en intensité, avec seulement 711034 changements de domicile? .

\section{La population rurale, moteur des migrations}

7 L'un des traits caractéristiques de la migration interne sous le socialisme tient au fait qu'elle a pour moteur la population agricole. Dans la décennie 1956-1965, trois-quarts des migrants sont d'origine rurale; au cours de la période suivante, leur part reste 
supérieure à $60 \%$. Les chercheurs bulgares en concluent que le déclin de la population rurale et, consécutivement, de sa participation aux mobilités internes, constitue le principal facteur de ralentissement des migrations dans les années $1980^{10}$. La place prépondérante de la population rurale dans les larges processus migratoires internes que la Bulgarie a connus au cours des années 1960 et 1970 se répercute également sur la direction des migrations (les rapports villages-villes), comme le montre le tableau 1.

Tableau 1 - Les migrations internes en Bulgarie entre 1956 et 1985 (en \%)

\begin{tabular}{|l|l|l|l|}
\hline Direction des migrations & $\mathbf{1 9 5 6 - 1 9 6 5}$ & $\mathbf{1 9 6 6 - 1 9 7 5}$ & $\mathbf{1 9 7 6 - 1 9 8 5}$ \\
\hline Du village vers la ville & 44,7 & 42,7 & 34,3 \\
\hline Village-village & 30,6 & 17,2 & 14,1 \\
\hline Ville-ville & 16,6 & 30,2 & 38,3 \\
\hline Ville-village & 8,1 & 9,9 & 13,3 \\
\hline
\end{tabular}

Source : NSI, Prebrojavane na naselenieto, (op.cit.), p. 28.

8 À la fin des années 1950 et au début des années 1960, les mouvements les plus importants vont du village vers la ville à l'unisson avec les processus d'urbanisation, d'industrialisation et de collectivisation des terres agricoles. Les déplacements intervillageois arrivent en seconde place : leur visibilité et leur importance proviennent du fait qu'à cette époque l'essentiel de la population bulgare réside encore en milieu rural. Au cours des années 1966-1975, l'exode rural continue à dominer, mais les mouvements inter-urbains se hissent au second rang en raison de l'augmentation rapide de la population urbaine. Entre 1976 et 1985, pour la première fois les déplacements villeville deviennent prépondérants dans la structure des courants migratoires, tandis que les déplacements intervillageois ou ville-village sont d'ampleur presque égale. Sur les quatre décennies socialistes, plusieurs tendances stables se dessinent ainsi dans l'orientation des migrations: l'exode rural décroît progressivement, tout comme les migrations de village à village, tandis que les mouvements inter-urbains s'accroissent, ainsi que ceux de la ville vers les villages, quoiqu'à des rythmes plus lents. L'accroissement des migrations inter-urbaines doit également à des changements strictement administratifs, c'est-à-dire à la transformation de certains villages en ville (et, jusqu'en 1968, à l'existence de villages de type urbain): en l'espace de quatre décennies, environ 130 de plus gros bourgs se sont ainsi vu octroyer le statut de villes. ${ }^{11}$

Entre 1946 et 1956, c'est la capitale, Sofia, qui enregistre les soldes migratoires les plus élevés, 12188 habitants par an en moyenne; suivent les villes de Plovdiv (3 072), Ruse (2 426) et Varna (2 050) (voir carte 1). Quelques autres centres urbains connaissent un solde migratoire supérieur à 1000 habitants: Burgas, Dimitrovgrad, Pernik, Pleven, Stara Zagora et Šumen. Šumen est le centre d'une région agricole élargie; Pernik et Dimitrovgrad sont des centres industriels à croissance rapide. Les autres villes appartenant à ce groupe sont des chefs-lieu de régions (oblasti) et plus tard de départements $(o k r a ̆ z i){ }^{12}$. Trois régions enregistrent le solde migratoire positif le plus élevé : la région de Sofia-Pernik, celle de Haskovo-Kărdžali et les villes de la Sredna Stara 
planina. Rares sont les agglomérations qui voient leur population se contracter durant cette période. Il s'agit avant tout de communes de montagne où l'approvisionnement de la population n'est pas toujours aisé et qui se situent à proximité d'importants centres industriels ${ }^{13}$.

Carte 1 - Les principales villes de Bulgarie (découpage administratif de 1999)

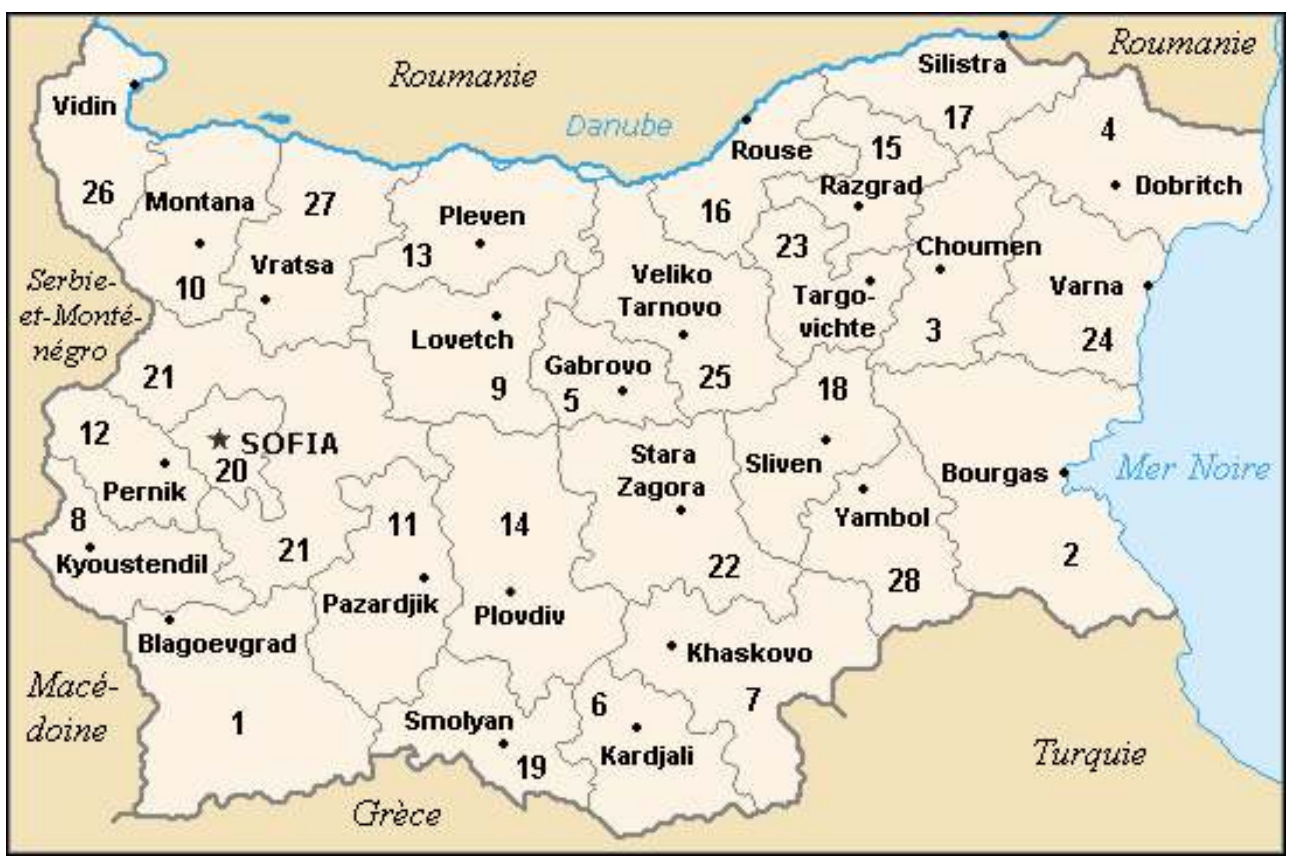

Source : http://commons.wikimedia.org/wiki/Image:Oblasti_de_Bulgarie_depuis_1999.png

\section{Le profil des migrants}

Pendant toute la période socialiste, la contribution des femmes aux mobilités internes excède celle des hommes. Entre 1946 et 1956, 130973 femmes ont migré pour seulement 105550 hommes. De 1956 à 1965, les femmes représentent 50,6\% des migrants ; elles sont 52,6\% entre 1966 et 1975 et leur part croît encore pour atteindre $54,1 \%$ entre 1976 et 1985 . Ce différentiel s'explique à la fois par la tradition qui veut que, lors d'un mariage, ce soit la femme qui change de lieu de résidence et par la participation active des femmes à la production et à la vie publique après $1944^{14}$. La majorité des migrants sont jeunes. Néanmoins, à partir de 1966, le taux des plus de 60 ans s'accroît progressivement: de 3,7\% en 1966-1975, il s'élève à 5,4\% au cours de la décennie suivante pour atteindre 9,4\% sur la période $1986-1992^{15}$. Les recensements de 1975 et de 1985 nous fournissent également des renseignements sur l'état civil des migrants. En 1975, les personnes mariées prédominent (53,8\% contre $41,9 \%$ de célibataires) ; mais le pourcentage de ces derniers au sein de la population globale est nettement inférieur à celui des mariés (34,7\% contre 57,7\%). En 1985, les célibataires représentent $34 \%$ de la population, mais ils contribuent à hauteur de $38,5 \%$ aux migrations, alors que les personnes mariées (56,3\%) ne comptent que pour $55,3 \%$ des migrants. Sur l'ensemble de la période socialiste, la tendance est à un léger déclin du pourcentage des personnes mariées en Bulgarie, tandis que leur participation aux migrations s'accroît faiblement. Une évolution très sensible s'observe également dans le profil scolaire des migrants. En 1956-1965, une large majorité des migrants (73,2\%) a 
un niveau d'éducation primaire ou secondaire (collège). Au cours de la décennie suivante, leur part tombe à $50,7 \%$, puis à $37,2 \%$ pour la période $1976-85$, reflétant la généralisation de l'accès à l'enseignement secondaire et les mutations sociales plus larges impulsées par le régime socialiste ${ }^{16}$.

11 Le profil type du migrant pendant la période socialiste en Bulgarie est une femme, paysanne, jeune, mariée, qui a arrêté ses études après l'école primaire ou le collège et travaille dans l'industrie. Cette tendance n'est pas propre aux États socialistes. En 1975 déjà, dans son analyse des migrations internes en URSS, Peter Grandstaff observait qu'en dépit des différences essentielles supposées entre la société soviétique et les sociétés à économie de marché, les processus migratoires des pays occidentaux décrivaient assez bien l'expérience soviétique des années 1960-1970. La grande ampleur des migrations en URSS était liée au développement industriel. La croissance de l'industrie provoquait des circulations internes, tandis que les niveaux soutenus de développement industriel engendraient d'importants déplacements inter et intrarégionaux. Le piètre prestige des métiers agricoles, l'attrait pour le travail et la vie en milieu urbain étaient à l'origine d'un fort exode rural. Peter Grandstaff concluait que les tendances migratoires des pays socialistes et capitalistes présentaient des ressemblances significatives et que, pour ce qui concernait les mobilités internes, les sociétés socialistes et de marché suivaient des trajectoires similaires ${ }^{17}$.

\section{Les politiques de gestion des mobilités sous le régime socialiste}

12 La politique gouvernementale active de gestion de la population constitue un autre facteur ayant significativement influencé les migrations internes en Bulgarie socialiste. Cette catégorie comprend - outre la distribution des permis de domiciliation - les politiques de peuplement des régions frontalières (en vue d'y contrôler la composition ethnique de la population) ou encore des zones à faible population (comme la région de Strandža-Sakar dans le sud-est de la Bulgarie), l'expulsion hors des villes des personnes au comportement jugé "amoral" et les efforts d'amélioration de la composition sociale de la capitale comme des grands centres régionaux ${ }^{18}$. Les démarches de l'État visant à créer une nouvelle ville socialiste - Dimitrovgrad, par exemple - et à en faire un symbole de la nouvelle société et du nouvel homme socialiste, ont également encouragé les migrations. En 1947 au moment où commence la construction de Dimitrovgrad, la population des trois villages qui forment son noyau est de 9200 âmes. En 1956, Dimitrovgrad compte 34200 habitants; elle atteindra environ 54000 habitants au milieu des années $1980^{19}$. Mais c'est sans doute l'interprétation, par le gouvernement, des migrations internes comme un processus planifié dont le but essentiel était de répondre aux besoins régionaux en main d'œuvre qui a joué le rôle principal dans la détermination de l'ampleur et dans l'orientation des mouvements de population. Les chercheurs qui ont étudié les politiques socialistes de gestion des populations ne manquent pas de souligner que la plupart d'entre elles étaient des "politiques ex ante", conçues et adoptées pour influencer, changer, améliorer le peuplement, non pour accompagner et réguler des événements et des processus en cours et que, le plus souvent, elles ont été subordonnées à une rationalité plus sociale que démographique ${ }^{20}$.

La transition politique et économique de l'après 1989 a mis un terme à ces politiques. Le contrôle strict des mobilités internes a été levé au mois d'août 1990, lorsque le premier Président démocratiquement élu de Bulgarie, Jeliou Jelev, a supprimé le régime de 
domiciliation. Il convient maintenant de déterminer si la disparition des régimes d'encadrement $d u$ peuplement en milieu urbain a exercé une influence sur les tendances migratoires en Bulgarie qui pourrait être comparée aux effets de la suppression du contrôle des frontières extérieures sur les migrations internationales. Les changements politiques, économiques et sociaux radicaux suscitent-ils de nouvelles dynamiques migratoires en Bulgarie après 1989?

\section{Les mobilités internes en Bulgarie après 1989 : explosion des déplacements après la levée des contrôles ou persistance des tendances antérieures ?} bulgare est restée largement inchangée après 1989. L'annulation des restrictions administratives pesant sur le libre choix du lieu de résidence n'a pas donné lieu à l'accroissement des mobilités que l'on aurait pu anticiper ${ }^{21}$. Entre 1986 et 1992, 345711 personnes ont changé de lieu de résidence et, pour la période 1993-2001, le nombre des migrants s'élève à 385 637, la différence entre les deux résultats étant certainement imputable non à une intensification de la mobilité interne mais à la période plus longue entre les deux recensements. Deux facteurs peuvent expliquer le ralentissement des migrations internes. L'absence d'un marché du travail suffisamment dynamique dans la plupart des régions de peuplement est le premier. En effet, dans la décennie qui fait suite aux changements de 1989, la nécessité d'une restructuration radicale de l'économie et des droits de propriété a entraîné une profonde crise économique, marquée par la fermeture de maintes entreprises industrielles (en particulier dans l'industrie lourde), une chute de la production agricole et un taux de chômage élevé. Entre 1989 et 1997, le PNB perd ainsi 32\%, tandis que le taux d'inflation annuel moyen sur la période 1990-1997 est de 210,1\% ${ }^{22}$. L'ouverture des nouvelles opportunités de migrations internationales constitue le second paramètre ayant pesé sur le dynamisme des migrations internes.

\section{Ressemblances et dissemblances dans les tendances migratoires de l'avant et après 1989}

La surreprésentation des femmes dans les mobilités internes se maintient. Pour la période 1986-1992, 52,9\% de personnes ayant changé de lieu de résidence sont des femmes. Leur part atteint 54,1\% entre 1993 et $2001^{23}$. Les jeunes continuent à être plus actifs dans les mobilités internes que leurs aînés : entre 1993 et 2001, plus de la moitié des migrants (57\%) ont moins de 29 ans. De la même façon, demeure inchangée la tendance à un accroissement de la part des plus de soixante ans: en 1986-1992, ils représentaient 9,5\% des migrants; en 1993-2001, leur nombre s'élève à 11,1\%.La diminution, entamée pendant la période socialiste, de la part de personnes ayant reçu une éducation primaire ou élémentaire parmi les migrants se poursuit au profit d'une hausse du nombre des diplômés du lycée et de l'enseignement supérieur. En 1986-1992, le pourcentage des migrants qui ont fait des études élémentaires et primaires est tombé à $35,2 \%$; il continue de se contracter sur la période $1993-2001$ pour atteindre $29,8 \%$. À l'inverse, en 1986-1992, la part des migrants ayant accédé à l'enseignement secondaire 
(lycée) ou supérieur (deug ou bachelor) dépasse pour la première fois les $50 \%$ pour culminer à près de $60 \%$ sur la période $1993-2001^{24}$.

On peut toutefois constater, dans le profil des participants aux mobilités internes, des tendances tout à fait nouvelles. À la fin des années 1980, la part des actifs dans les migrants a brutalement chuté pour tomber en deçà de $45 \%$; vers l'année 2001, elle s'était légèrement redressée à $48,1 \%$. La frange des actifs au sein de la population migrante s'est ainsi révélée légèrement inférieure à leur pourcentage dans la société, lequel s'élevait en 2001 à $48,6 \%{ }^{25}$. En toute probabilité, ce phénomène plonge ses racines dans les restructurations radicales qui ont affecté tous les secteurs de l'économie et le marché du travail dans l'ensemble du pays après 1989. Bien que la tendance à la réduction, parmi les migrants, des employés dans l'industrie soit une constante depuis le milieu des années 1960, en 1993-2001, ils restaient le groupe migrant le plus important avec $21,2 \%$. On observe en revanche une forte hausse du nombre des migrants travaillant dans le commerce, l'hôtellerie, la restauration et les travaux de réparation (19,3\%, en deuxième place) à l'image de la recomposition des secteurs d'activité économique observée en Bulgarie au cours de ces années de transition vers une économie de marché à forte insertion internationale ${ }^{26}$.

17 Les actifs qui effectuent quotidiennement des trajets plus ou moins longs pour se rendre sur leur lieu de travail représentent une catégorie intéressante ${ }^{27}$. Pendant la période socialiste, leur nombre avait augmenté au fur et à mesure que se développaient, dans de petites et moyennes communes, des industries fournissant un emploi aux populations des villages environnant. Une enquête sociologique réalisée parmi 3819 ruraux en 1988 avait ainsi établi qu'un quart d'entre eux parcouraient chaque jour plus de 4 kilomètres pour se rendre à leur travail ; mais que deux tiers d'entre eux n'effectuaient pas plus de 15 kilomètres. 8,6\% des participants à l'enquête avaient déclaré réaliser plus de 15 kilomètres par jour jusqu'à leur travail ${ }^{28}$. Après 1989, cette tendance s'est inversée. En 1975, les migrations de travail journalières concernaient 622716 personnes, en 1985, 751654 et seulement 328762 en 2001. Ce résultat est deux fois moins important qu'en 1975. Actuellement, $60 \%$ des participants aux migrations de travail journalières sont des hommes ( $40 \%$ des femmes). Environ $11 \%$ (37 698) des actifs se rendent tous les jours en dehors de leur département (oblast) pour travailler. Près de $61 \%$ des migrations de travail quotidiennes se réalisent dans des villes et $28 \%$ dans des villages du département de résidence des migrants. La majorité des flux concerne des personnes qui ont fait des études secondaires spécialisées; viennent ensuite les migrants ayant reçu une éducation primaire. Les diplômés de l'enseignement supérieur semblent moins mobiles, sans doute en raison de la concentration de ces derniers dans les centres urbains. Dans les villages, 17\% d'entre eux doivent se déplacer chaque jour ( $11 \%$ dans les villes). Les migrations les plus intenses sont enregistrées dans les départements de Sofia, Kjustendil, Pernik et Stara Zagora; à l'autre extrémité de l'échelle, se situent les départements de Vidin, Ruse, Montana ainsi que la ville de Sofia.

L'essentiel des migrations de travail quotidiennes dans la région de Sofia et les régions voisines de Kjustendil et de Pernik s'effectue en direction de la capitale, Sofia, qui constitue, dans le pays, le centre le plus attractif pour la main d'œuvre. Cette attractivité explique également la faiblesse du nombre des personnes allant chaque jour quérir un travail en dehors de la région de Sofia. Stara Zagora attire des migrants en raison du fort développement de l'industrie dans cette région, avec, notamment, le 
complexe industriel-énergétique Marica-Iztok, le producteur de bière Zagorska, du Domaine Menada viticole, etc. Les régions de Vidin et de Montana, toutes deux situées au nord-ouest de la Bulgarie, sont parmi les moins développées sur le plan économique, suite à l'effondrement de l'industrie chimique bulgare après 1989. Elles tendent également à voir leur poids démographique diminuer en raison du vieillissement de la population régionale, du taux de chômage élevé, de problèmes écologiques et d'une infrastructure de transport insatisfaisante. Mais, de façon générale, les circulations quotidiennes liées au travail n'ont pas entraîné, après 1989, de redistribution territoriale ou structurelle significative de la main d'œuvre ${ }^{29}$.

Des changements sont intervenus dans la situation familiale des migrants : la part des célibataires a augmenté au détriment de celle des mariés. En 1992, 43,9\% des migrants étaient non mariés et $49,2 \%$ mariés. En 2001, pour la première fois, le pourcentage des célibataires a dépassé celui des personnes mariées (respectivement $49,4 \%$ et $40,5 \%)^{30}$. Ce renversement s'explique certainement par les nouvelles dynamiques migratoires contemporaines : les changements de résidence à des fins matrimoniales sont passés à l'arrière-plan, tandis que les mouvements liés à la recherche d'un emploi et au marché du travail ont acquis une visibilité et une importance nouvelles ${ }^{31}$. Le développement de formes de vie commune en dehors du mariage peut également avoir exercé une influence, tout comme les mariages plus tardifs. Il n'est pas sans intérêt, pour cette raison, de citer les résultats d'une enquête sociologique représentative consacrée à l'analyse des migrations de la décennie passée en Bulgarie : près de $50 \%$ des personnes interrogées ont indiqué qu'elles avaient entrepris une mobilité pour des raisons familiales; $37 \%$ ont invoqué comme motivation la recherche d'un emploi qui corresponde à leur qualification professionnelle ${ }^{32}$.

\section{De la ville vers le village}

Plusieurs traits saillants ressortent également de l'observation de la direction des flux migratoires détaillée dans les tableaux 2 et 3.

Tableau 2 - Les migrations internes en Bulgarie entre 1956 et 1985 (en \%)

\begin{tabular}{|l|l|l|}
\hline Direction des migrations & $\mathbf{1 9 8 6 - 1 9 9 2}$ & $\mathbf{1 9 9 3 - 2 0 0 1}$ \\
\hline Du village vers la ville & 22,0 & 16,6 \\
\hline Village-village & 12,1 & 10,8 \\
\hline Ville-ville & 42,5 & 46,5 \\
\hline Ville-village & 23,4 & 26,1 \\
\hline
\end{tabular}

Source : NSI, Prebrojavane na naselenieto, op. cit., p.28.

21 Le tableau 2 montre une persistance des dynamiques de la période socialiste, avec une réduction graduelle de l'exode rural et des migrations intervillageoises et un accroissement des déplacements interurbains, ainsi que des mobilités entre les espaces urbains et ruraux. La structure actuelle de la population en Bulgarie, urbaine à $69 \%$, 
explique en partie la nature de ces mouvements; d'autres raisons tiennent aux opportunités d'emploi, aux prix du logement, aux niveaux de vie, etc. Les données disponibles mettent par ailleurs en évidence plusieurs processus inédits, telle la prépondérance des migrations villes-villages. À la fin $\mathrm{du} \mathrm{XX}^{\mathrm{ème}}$ siècle, ces dernières apparaissent une fois et demi supérieures aux mouvements en sens inverse.

Tableau 3 - Les migrations internes en Bulgarie en 2004 et 2005 (en \%)

\begin{tabular}{|l|l|l|}
\hline Direction des migrations & $\mathbf{2 0 0 4}$ & $\mathbf{2 0 0 5}$ \\
\hline Village-ville & 21,0 & 22,0 \\
\hline Village-village & 11,0 & 10,0 \\
\hline Ville-ville & 45,0 & 44,0 \\
\hline Ville-village & 23,0 & 24,0 \\
\hline
\end{tabular}

Source : NSI, Naselenie i demografski procesi prez 2005

En 2004 et 2005 (tableau 3), la répartition des migrations internes est demeurée inchangée : en 2004, environ 145000 migrations ont été enregistrées dans le pays, qui ont concerné 137000 personnes $^{33}$. Les données pour l'année 2005 sont de 168000 (environ 150000 migrants). Les déplacements les plus nombreux suivent une ligne ville-ville, suivis par les mobilités villes-villages. Au terme de ces mouvements entre espaces urbains et ruraux, la population urbaine a décru de 3164 personnes en 2005, un chiffre qui a pour pendant un accroissement de l'habitat rural ${ }^{34}$.

Les politiques de restitution des terres agricoles ${ }^{35}$, le chômage et l'attractivité du travail agricole (grâce aux subsides accordés par l'Union européenne au titre du processus d'adhésion) $)^{36}$, ainsi que les problèmes relatifs à la réforme du système de retraites ${ }^{37}$ éclairent ces retours au village. Le phénomène n'est d'ailleurs pas propre à la Bulgarie post-socialiste. Des processus similaires ont été observés en Roumanie voisine après 1997 pour des raisons identiques à celles rencontrées en Bulgarie - le chômage en milieu urbain et la restitution des terres, etc. ${ }^{38}$. En termes générationnels, les 50-59 ans constituent le premier groupe de migrants (22\%) devant les $40-49$ ans $(16 \%)^{39}$. Autrement dit, les personnes qui quittent les villes pour le monde rural ne sont pas seulement des retraités, désireux de passer leurs dernières années dans un univers rural serein, propre et calme, mais des personnes en âge de travailler qui espèrent, par ce changement de résidence, trouver les moyens de gagner leur vie.

D'un point de vue régional, les migrations internes de l'après 1989 n'ont pas suivi de direction clairement définie, à la différence, par exemple, de ce qui a pu être observé en ex-URSS où les mobilités intervenues après 1991 (dorénavant des migrations externes entre les nouvelles Républiques souveraines) se sont caractérisées par un ample mouvement depuis l'Ouest vers l'Est ${ }^{40}$. Sous l'effet des déplacements régionaux en Bulgarie, en 2001, 21 départements (sur 28) avaient vu leur population diminuer de 61843 personnes au total. Les départements de Smoljan (dans les Rhodopes occidentaux), de Silistra (au nord-est du pays), de Vraca et de Montana (nord-ouest), de Haskovo (Rhodopes orientaux), de Tărgovište (nord-est) et de Jambol (centre-est) 
étaient particulièrement touchés, principalement en raison de la dégradation de la situation économique. Seuls sept départements présentaient un solde migratoire positif. Sofia détenait la première place avec 52347 nouveaux habitants, suivie par Varna, Plovdiv, Veliko Tărnovo, Šumen, Burgas et Gabrovo. Les raisons de ces mouvements sont à rechercher, notamment, dans le développement de la construction et du tourisme autour de la mer Noire, la prospérité économique de Plovdiv et le développement de ces villes en tant que centres universitaires. Entre 1993 et 2001, quelque $42 \%$ des migrations sont intervenues à l'intérieur même des départements. Le flux qui se dessine le plus clairement concerne les mouvements en direction de Sofia, centre migratoire le plus attractif. Les autres dynamiques notables portent sur des déplacements entre départements voisins. Dans $60 \%$ des cas, les actifs franchissent les frontières de leur département d'origine ${ }^{41}$.

Avec la création d'un système de planification régional, en 2000, sous l'influence du processus de préparation de l'intégration à l'Union européenne, a commencé la collecte de données relatives aux mobilités au sein comme entre les six régions de planifications NUTS (sud-ouest, sud-centre, sud-est, nord-ouest, nord-centre, et nord-est) ${ }^{42}$. Seule la région sud-ouest (qui comprend la capitale) a enregistré un solde migratoire positif entre 2001 et 2003, tandis que les cinq autres voyaient leur population décroître sous l'effet des migrations internes ${ }^{43}$.

\section{Les migrations ethniques}

Les recensements de 1992 et de 2001 nous permettent de mieux connaître l'appartenance ethnique des personnes mobiles. Dans les deux cas, la mobilité des représentants des principaux groupes ethniques correspond globalement à leur poids démographique au sein de la population. Sur la période 1992-2001, les Bulgares représentent la part la plus importante des migrants $(81,9 \%)$, suivis par les Turcs $(9,5 \%)$ et les Roms $(4,1 \%)^{44}$. Dans le même temps, seuls $55 \%$ des Roms de Bulgarie vivent en milieu urbain, alors que la population urbaine représente en moyenne plus de $69 \%$ de la population du pays. ${ }^{45}$ La concentration des Roms dans les villages, leur faible maîtrise de la langue bulgare et leur difficulté à communiquer dans un environnement dynamique constituent des variables importantes dans la décision de migrer à l'intérieur du pays et semblent s'accompagner d'une moindre participation des Roms de Bulgarie aux migrations internes. Couplé avec l'abandon des villages par leurs autres habitants, le grand nombre des Roms vivant en milieu rural est en train de transformer certaines écoles en écoles de facto rom - un problème inédit pour la société et le système scolaire bulgares ${ }^{46}$. On rappellera toutefois que les données statistiques disponibles concernant les populations roms sont peu fiables - que ce soit en raison de leur identification à un autre groupe ethnique ou parce qu'ils ne sont pas enregistrés - et il est possible que leurs mobilités ne soient pas correctement appréciées.

Bien que les Roms ne soient pas statistiquement surreprésentés dans les circulations internes, leurs mouvements reçoivent souvent une attention médiatique et sociétale supérieure à celle des Bulgares et des Turcs. Il n'est pas rare que les projets d'installation de familles ou de groupes roms dans de nouveaux villages, villes ou quartiers de la capitale se heurtent à une forte hostilité et aux protestations des habitants. La concentration de la population rom dans certains quartiers de Sofia ainsi que dans les grandes villes est souvent interprétée comme reflétant, chez les Roms, une 
stratégie de sélection des lieux de résidence en fonction des opportunités d'accès aux aides sociales. Le déplacement des Roms vers des sites où l'Etat prévoit de leur construire des logements ou encore, s'ils sont nouveaux venus, leur rapatriement vers leur lieu de domiciliation permanent sont parfois vus comme les réponses à apporter aux problèmes sociaux auxquels ils sont confrontés : la pauvreté, les constructions illégales de logements ou encore la criminalité.

\section{Les politiques gouvernementales de gestion des migrations}

Si les politiques socialistes de gestion des migrations intérieures ont été supprimées à la fin 1989, les migrations internes n'ont pas pour autant été confiées à la seule logique du marché. Après 1989, les gouvernements bulgares (en particulier ces quatre ou cinq dernières années sous l'influence de la conditionnalité européenne) ont conçu une variété de programmes visant à administrer les migrations de travail. En lieu et place des anciennes politiques socialistes ont été développées des politiques destinées à assurer un développement plus équilibré des régions et à réduire le taux de chômage en augmentant la mobilité de la main d'œuvre. Depuis 1989 et, plus encore, depuis 2001, l'État bulgare n'envisage nullement de renoncer à réguler les migrations internes, lui qui a entièrement repris à son compte la vision - présente au sein de l'EU - selon laquelle la mobilité de la main d'œuvre constituerait un facteur-clé de croissance économique et d'augmentation des niveaux de vie.

Toute une série de documents du ministère du Travail et des Affaires sociales (à l'image des plans d'action nationaux pour l'emploi de 2005 et 2006) se donne pour priorité l'intensification de la mobilité et de la flexibilité des ressources humaines. L'accroissement de l'adaptabilité, de la mobilité et de la compétitivité de la main d'œuvre figure ainsi parmi les principales priorités de l'actuel Programme opérationnel pour "le développement des ressources humaines" du ministère des Affaires sociales ${ }^{47}$ qui fournit le cadre de la gestion des fonds structurels européens pour la période allant de 2007 à 2013. La stratégie bulgare pour l'emploi 2004-2010, adoptée par le Conseil des ministres en novembre 2003 et préparée conformément à la stratégie européenne en matière d'emploi, a entièrement repris à son compte les dix priorités de cette dernière, parmi lesquelles figurent l'encouragement à l'adaptabilité et à la mobilité sur le marché du travail ${ }^{48}$.

\section{Etudier en parallèle les migrations internes et externes : un nouvel agenda de recherche}

Ces dernières années, les auteurs spécialisés dans l'étude des migrations - en particulier transnationales - ont remis en cause l'un des principes fondateurs de la recherche en sciences sociales, à savoir les délimitations territoriales. Les études des trans-migrants montrent comment ces derniers parviennent à entretenir des réseaux sociaux multiples aussi bien avec leur pays d'origine qu'avec l'État qui les a récemment accueillis en franchissant aisément les frontières nationales et en parvenant à contourner les institutions classiques de l'État-nation. Le concept, d'ores et déjà largement usité, d'État-nation déterritorialisé aide à diluer les frontières nationales et à dépasser le modèle de la société-container. La critique du nationalisme méthodologique 
dans les sciences sociales remet en question la conviction selon laquelle l'État-nation serait la seule forme politique et sociale du monde moderne ${ }^{49}$.

Dans ces conditions, il est étonnant que, précisément dans ces études pionnières qui ont ébranlé la stabilité de l'État-nation comme catégorie d'analyse, une stricte séparation entre les migrations internes et externes prédomine. Non que soient totalement absentes les études abordant conjointement les deux types de migrations, mais leurs processus caractéristiques sont considérés comme distincts. Une telle démarche cimente la centralité de la frontière étatique et empêche de découvrir le degré de dépendance mutuelle entre les déplacements à l'intérieur du territoire de l'État-nation et en dehors de lui. Le travail de Neda Deneva constitue une exception à cette règle. Dans l'une des études les plus innovantes et les plus riches consacrées aux migrations bulgares contemporaines, elle a établi - en s'appuyant sur l'exemple du village de Ribnovo, au Sud-Ouest de la Bulgarie- un lien entre les mouvements intervenant à l'intérieur de la Bulgarie et en dehors de ses frontières ${ }^{50}$.

Dans le cas bulgare, une démarche particulièrement heuristique consisterait à étudier la manière dont les migrations internationales - au sein desquelles prédominent les formes de migrations temporaires, saisonnières, circulaires et par réseaux au détriment des émigrations permanentes - influencent les intentions et décisions de circulation à l'intérieur du pays, à la faveur de retours de courte durée, de moyenne durée ou définitifs. Les études disponibles montrent en effet que de telles mobilités internes sont provoquées et aiguillées par les migrations externes qui les ont précédées. Il serait utile d'étudier quel facteur ou quelle combinaison de facteurs suscite les déplacements internes : l'épargne migrante, les savoir-faire acquis ou le changement culturel lié à la nouvelle organisation du travail et au nouveau mode de vie, source de nouvelles attitudes et visions du monde ${ }^{51}$. Il serait également intéressant de déterminer le rôle des moyens financiers, ramenés en Bulgarie ou envoyés à des parents, dans l'encouragement des migrations internes depuis les villages vers les villes, depuis les centres urbains vers les espaces ruraux ou dans toute autre direction. Au nord-est de la Bulgarie, la migration saisonnière des Turcs bulgares vers la Suède, l'Allemagne, la Belgique, la Hollande et l'Espagne a entraîné d'autres migrations aussi bien à l'intérieur qu'à l'extérieur du pays. Dans la région de Kărdžali (Rhodopes orientaux), s'observe par exemple un déplacement des Turcs d'abord des villages vers les villes proches, puis, vers les grands centres urbains et les chefs-lieu de district. Ces mouvements sont renforcés par l'épargne issue des migrations saisonnières à l'étranger. Dans un premier temps, ces ressources sont utilisées pour réparer les maisons au village, puis pour acheter des biens fonciers à Kărdžali et enfin pour acquérir un appartement à Plovdiv, la seconde plus grande ville de Bulgarie. Une fois les économies investies dans une propriété, les migrants saisonniers restent sans emploi en Bulgarie ; ils ne souhaitent pas travailler pour un salaire inférieur à celui auquel ils ont été habitués à l'étranger et n'ont pas la possibilité de lancer leur propre activité entrepreneuriale. Cette situation les contraint à entamer un nouveau cycle de migrations temporaires, saisonnières ou circulaires en dehors du pays.

Plus fructueuse encore serait l'étude de la manière dont les migrations internationales peuvent conduire à une immobilité contrainte dans des secteurs économiques à forte mobilité traditionnelle comme la construction, par exemple. En raison du caractère propre à ce type de travail, les ouvriers du bâtiment figurent parmi les groupes professionnels les plus mobiles depuis l'époque du régime socialiste. Après 1989, ils 
sont devenus une des mains d'œuvre les plus recherchées à l'étranger. Légalement ou au noir, les ouvriers bulgares (en particulier les Turcs et les Pomaks, mais aussi les Bulgares ethniques) trouvent généralement un emploi en Allemagne, en Autriche, en Belgique, aux Pays-Bas, en Grèce ou ailleurs. Cependant, à leur retour en Bulgarie, en dépit du développement rapide de la construction ces dernières années, du déficit permanent de la main d'œuvre dans ce secteur et des rémunérations relativement attractives qui y sont proposées (par comparaison avec les autres domaines d'activité en Bulgarie), ils peinent à trouver du travail, démotivés par l'absence de salaires "européens". Il en résulte fréquemment une immobilisation forcée - une inertie imposée à soi-même - dans l'attente de nouvelles opportunités de départ à l'étranger.

Un autre domaine de recherche prometteur concerne les mouvements internes des immigrants en Bulgarie, qu'ils soient des citoyens étrangers ayant trouvé un refuge, une famille ou un travail dans le pays ou des citoyens bulgares revenus après un long séjour à l'étranger. Par comparaison avec celles des populations locales, la migration interne des étrangers n'a attiré l'attention que d'un faible nombre de chercheurs ${ }^{52}$. Il conviendrait pourtant d'étudier quelle contribution les immigrants installés en Bulgarie apportent aux migrations internes. La remarque vaut pour les immigrants économiques (par exemple, les Chinois dans le secteur de la restauration ou du commerce à un lev) comme pour les migrants qui s'installent en Bulgarie en raison du climat, de l'environnement et de la différence de niveaux de vie. Ces dernières années, le développement d'une nouvelle migration de retraités - principalement des Anglais, des Irlandais et des Japonais - a pu être observé. Selon certains analystes, ces flux devraient s'imposer dans les décennies à venir comme l'un des courants migratoires les plus importants au sein de l'Europe unie ${ }^{53}$. En raison de la stratégie d'installation en groupe de ces migrants ou de leur implantation dans certains villages situés au bord de la mer Noire ou en montagne, il serait intéressant de déterminer si leur présence suscite également l'arrivée de Bulgares attirés par la perspective d'une redynamisation du tissu économique des régions concernées grâce à l'apport d'argent étranger.

\section{Conclusion}

L'étude de la migration interne en Bulgarie a montré que plusieurs tendances importantes de la période socialiste s'étaient maintenues après les grandes transformations politiques, économiques et sociales de 1989. Parmi celles-ci figurent la diminution des flux migratoires depuis les années 1960 et le déclin de l'exode rural. Le sexe, l'âge et le niveau d'éducation des participants aux mobilités sont restés largement inchangés. De ce point de vue, 1989 ne représente pas une ligne de partage et une rupture marquée. Dans le même temps, cependant, le libre franchissement des frontières bulgares, l'intégration à l'Union européenne et la nouvelle place de la Bulgarie au sein de la communauté internationale ont exercé une influence significative sur les migrations internes. Envisager ces processus dans leurs interrelations avec les migrations internationales représente le plus grand défi pour l'étude des migrations en Bulgarie aujourd'hui. 


\section{NOTES}

1. En mars 1948, l'État bulgare avait adopté une loi sur la nationalité envisageant la possibilité de déchoir de leur nationalité des Bulgares ayant voyagé hors du pays sans autorisation. De 1944 à 1948, le contrôle des sorties du territoire relevait des prérogatives de la Commission de contrôle interalliée, qui a également mis en œuvre une politique de déplacement de la population en Bulgarie et émettait les autorisations d'entrée comme de sortie du pays. Voir Büchsenschütz (Ulrich), Malcinstvenata politika v Bălgarija. Politikata na BKP kăm evrei, romi, pomaci i turci (1955-1989) [La politique des minorités de la Bulgarie. La politique du Parti communiste bulgare envers les juifs, les Roms, les Pomaks et les Turcs (1944-1989)], Sofia : IMIR, 2000, pp.13, 95, à l'adresse : http://www.imir-bg.org/imir/books/malcinstvena\%20politika.pdf. Le 4 mars 1948, l'État bulgare a adopté la Loi sur les passeports et le contrôle de la milice sur les étrangers, qui introduisait des visas de sortie ("miliciens") et interdisait l'attribution de passeports aux citoyens "dont les voyage é[tait] inutile ou nuisible à l'État ». Deux jours plus tard était adoptée la Loi sur la citoyenneté bulgare qui permettait d'ôter aux personnes ayant illégalement quitté le pays leur citoyenneté bulgare et de confisquer leurs biens au profit de l'État. Voir Dăržaven vestnik, (69), 25/03/48 et (70), 26/03/48. En 1951, les sorties illégales de territoire ont été criminalisées avec l'introduction dans le code pénal d'un crime de "préparation, tentative et fuite hors des frontières ", passible de dix années d'emprisonnement et de 500000 leva d'amende (ces deux clauses ont été par la suite adoucies à divers degrés). Voir Nakazatelen zakon [Code pénal], Sofia : Nauka i izkustvo, 1952; Nakazatelen kodeks [Code pénal], Sofia: Nauka i izkustvo, 1956; Nakazatelen kodeks [Code pénal], Sofia: Nauka i izkustvo, 1969. Les proches des "émigrés" pouvaient eux aussi faire l'objet de poursuites : en vertu de la loi, leur correspondance pouvait être ouverte et les biens des époux restés dans le pays pouvaient être confisqués par l'État. En dehors des mesures prévues par la loi, les parents des personnes ayant fui à l'étranger ont souvent fait l'objet d'une surveillance par les organes de la Sûreté d'État (Dăržavna sigurnost); voyager à l'étranger (et en particulier à l'Ouest) leur était interdit; ils ont souvent été soumis poursuivis et exilés (à l'intérieur du pays) et leur progression de carrière a été entravée.

2. Le 11 mai 1989, les pouvoirs bulgares ont amendé et complété la Loi sur les passeports internationaux sous la pression de l'effervescence enregistrée au sein des populations de Turcs bulgares - soumises depuis 1984 à une politique d'assimilation forcée connue sous le nom de "Processus de renaissance". Le nouvel article 1 a posé que tous les citoyens bulgares avaient le droit de quitter le pays et d'y revenir, alors que les ordonnances antérieures prévoyaient que «les citoyens de République populaire de Bulgarie peuvent franchir la frontière étatique et séjourner dans un autre pays ». Dans le même temps, cependant, les visa de sortie ont été maintenus; toutefois, un délai d'un mois a été introduit pour l'examen des demandes de passeport et de visa, tout refus devant désormais être motivé par écrit avec la possibilité, pour le requérant, de faire appel de la décision. L'amende prévue en cas d'infraction au régime des passeports est passée de 200 à 500 leva, pouvant atteindre 1000 leva en cas de retour dans le pays deux ans après l'expiration du visa de sortie. Cette dernière clause, tout comme le postulat selon lequel les personnes devant des sommes significatives à l'État ou à d'autres citoyens ne peuvent se voir remettre un passeport, montrent que les changements introduits dans le régime de sortie du pays ont été conçus en vue d'une future émigration des Tucs bulgares vers la Turquie. Bien que le nouveau texte ait prévu une entrée en vigueur des amendements au $1^{\text {er }}$ septembre 1989 , le gouvernement a commencé à émettre des passeports et des visas pour les Turcs bulgares immédiatement, rendant possible l'émigration massive de l'été 1989, appelée, par euphémisme, "la grande excursion". Voir Dăržaven vestnik, (38), 19/05/89. La majorité des citoyens bulgares n'a 
réellement obtenu la possibilité de sortir et d'entrer dans le pays qu'après le renversement de Todor Jivkov, le 10 novembre 1989.

3. Selon les données officielles du dernier recensement de 2001, 204600 Bulgares vivraient en Ukraine. Voir http://www.ukrcensus.gov.ua/eng/results/general/nationality/. Le dernier recensement de Moldavie, qui date de 2004, suggère la présence de 65662 Bulgares dans ce pays (mais ce chiffre en comprend pas les Bulgares de Transnistrie, lesquels sont estimés par l'Agence pour les Bulgares à l'étranger à plus de 13 000). Voir http://www.statistica.md/recensamint.php? lang=ru et http://www.aba.government.bg/news.php?newsid=174. Selon des données officieuses, un grand nombre de Bulgares résideraient également aux États-Unis (plus de 200000 ), en Espagne (plus de 120 000) et en Grèce (110 000). Voir Nacionalnata strategija na Republika Bălgarija po migracija $i$ integracija (2008-2015) [Stratégie nationale de la République de Bulgarie pour les migrations et l'intégration], à l'adresse :http://www.mlsp.government.bg/bg/docs/index.htm.

4. Voir les articles consacrés aux migrations dans la revue, Naselenie [Population], après 1990.

5. Voir Appadurai (Arjun), Modernity at Large. Cultural Dimensions of Globalization, Minneapolis / London: University of Minnesota Press, 1996 ; Basch (Linda), Glick Schiller (Nina), Szanton Blanc (Cristina), Nations Unbound. Transnational Projects, Postcolonial Predicaments and Deterritorialized Nation-States, Pennsylvania: Gordon and Breach, 1994; Kivisto (Peter), "Theorizing transnational immigration: a critical review of current efforts ", Ethnic and Racial Studies, 24 (4) ; Bauböck (Rainer), "Towards a Political Theory of Migrant Transnationalism », International Migration Review, 37 (3), 2003 ; Werbner (Pnina), Modood (Tariq), eds., Debating Cultural Hybridity. Multi-cultural Identities and the Politics of Anti-Racism, London / New Jersey : Zed Books, 1997 ; Pratt (Mary Louise), Imperial Eyes: Travel Writing and Transculturation, London / New York : Routledge, 1992.

6. Voir Guentcheva (Rossitza), From Banishment to Ascribed Residence: Controlling Internal Movement in Socialist Bulgaria (1944-1989), Research Paper, Sofia: Center for Advanced Studies (CAS), 2006, à l'adresse : http://v3.cas.bg/cyeds/downloads/ From_Banishment_to_Ascribed_Residence.pdf [consultée le 25 août 2008].

7. Voir Vasileva (Bojka), Migracionni procesi v Bălgarija sled Vtorata svetovna vojna [Les processus migratoires en Bulgarie après la seconde Guerre mondiale], Sofia : Université Kliment Ohridski, 1991, p.106.

8. Ce boom migratoire n'a pas automatiquement suscité le développement de recherches sur ces thématiques. À la fin des années 1960 une étude systématique des tendances migratoires internes et de leurs conséquences pour la société bulgare faisait encore défaut. Un manque d'intérêt similaire pouvait être observé en URSS où, entre 1930 et 1964, aucun livre n'a été consacré aux mobilités internes. Voir Grandstaff (Peter), «Recent Soviet Experience and Western "Laws" of Population Migration », International Migration Review, 9 (4), 1975, p.479. À la fin des années 1960, l'étude des migrations en URSS a été proclamée prioritaire pour l'économie nationale, ce qui a donné une impulsion aux études universitaires non seulement en URSS, mais également dans l'ensemble du bloc socialiste. En Bulgarie, c'est en 1972 qu'ont paru les travaux de Minkov (Minko), Migracija na naselenieto [Migration de la population], Sofia : Partizdat, 1972 et de Totev (A.), Demografija. Izbrani temi [Démographie. Thèmes choisis], Sofia : UI "Kliment Ohridski", 1972. Ils ont été suivis deux ans plus tard par l'étude fondatrice Demografija na Bălgarija [Démographie de la Bulgarie], Sofia : Nauka i izkustvo, 1974.

9. Voir NSI (Nacionalen Statističeski Institut), Prebrojavane na naselenieto, žilištnija fond $i$ zemedelskite stopanstva prez 2001 [Recensement de la population, du fonds pour le logement et de l'agriculture en 2001], t.1; NSI, Naselenie [Population], livre 6; NSI, Migracija na naselenieto [Migration de la population], Sofia : NSI, 2004, pp.13-14.

10. Ibid.

11. Voir Živkova (Veska), Seloto. Sociologičeski analiz [Le village. Analyse sociologique], Sofia: Partizdat, 1989, p.9. 
12. Entre 1949 et 1959 , le maillage administratif de la Bulgarie reposait sur l'existence de départements (okrăzi, dont le nombre a oscillé entre 13 à 15 selon les années, la ville de Sofia jouissant du statut d'okrăg), eux-mêmes subdivisés en districts (okolij). De 1959 à 1987, le pays a été administrativement divisé en 28 départements, d'environ 300000 habitants chaque, et les okolij supprimés. Le nouveau découpage administratif adopté en 1987 comprenait 9 régions (oblasti), subdivisées en municipalités (obštini). Depuis 1998, le nombre des régions est passé de 9 à 28 - lesquelles correspondent aux départements de l'avant 1987 - tandis que le niveau communal a été maintenu (on comptait 265 communes en 2005).

13. Voir Vasileva (Bojka), op.cit., pp.100-101.

14. Ibid. ; voir aussi NSI, Prebrojavane na naselenieto, (op.cit.), pp.15-16.

15. Ibid., p.16.

16. Ibid., p.20.

17. Voir Grandstaff (Peter), art.cit., p.495 ; voir aussi Grandstaff (Peter), Interregional Migration in the USSR: Economic Aspects, 1959-1970, Durham : Duke University Press, 1980. Dans cet ouvrage, l'auteur développe l'idée selon laquelle un marché du travail relativement libre aurait existé dans l'URSS de l'époque. La thèse de Grandstaff selon laquelle le pouvoir soviétique aurait supprimé une partie des limites posées à la mobilité interne - ce qui aurait conduit à une réduction de la migration contrôlée et régulée par l'État - a été critiquée au début des années 1980 par des chercheurs n'acceptant pas les thèses relatives à la similitude des processus économiques et sociaux en URSS et dans les pays occidentaux. Pour une critique de ce type, voir Linz (Susan), « Review of Interregional Migration in the U.S.S.R.: Economic Aspects, 1959-1970. By Peter J. Grandstaff. Durham : Duke University Press, $1980 »$, Journal of Economic History, 41 (2), 1981.

18. Voir Guentcheva (Rossitza), op.cit.

19. Brunnbauer (Ulf), "The Town of the Youth": Dimitrovgrad and Bulgarian Socialism", Ethnologia Balkanica, 9, 2005, p.95. Entre 45 et 50000 membres des Brigades de jeunesse ont également participé à la construction de Dimitrovgrad. De la sorte, à partir de la fin des années 1940 le mouvement des Brigades de jeunesse en Bulgarie - auquel ont pris part près d'un demimillion de jeunes - s'est transformé en un instrument puissant de mise en mobilité et de mélange des populations. Voir ibid., p. 94.

20. Voir Macura (Milos), "Population Policies in Socialist Countries of Europe ", Population Studies, 28 (3), 1974, pp.373-374.

21. Voir NSI, Prebrojavane na naselenieto..., (op.cit.), p.14.

22. Voir Anatomija na prehoda. Stopanskata politika na Bălgarija ot 1989 do 2004 [Anatomie de la transition. La politique économique de la Bulgarie de 1989 à 2004], Sofia : Siela, 2004. Pour une chronologie politique de la transition en Bulgarie, voir Kalinova (Evgenija), Baeva (Iskra), Bălgarskite prehodi, 1939-2002 [Les transitions bulgares, 1939-2002], Sofia : Paradigma, 2002 ; voir aussi Rajčev (Andrej), Stojčev (Kănčo), Kakvo se sluči. Razkaz za prehoda v Bălgarija, 1989-2004 [Ce qui s'est passé. Récit de la transition en Bulgarie, 1989-2004], Sofia: IK “Iztok-Zapad”, 2004 et Čalăkov (Ivan) et al., Mrežite na prehoda : kakvo svăštnost se sluči v Bălgarija sled 1989 [Les réseaux de la transition : ce qui s'est réellement passé en Bulgarie après 1989], Sofia : IK “Iztok-Zapad”, 2008. 23. NSI, Prebrojavane na naselenieto..., (op.cit.), p.15.

24. Ibid., p.16.

25. Ibid., p.20.

26. Ibid., p.23.

27. Quelques chercheurs, qui se rallient à la définition officielle des migrations (comme absence de son lieu d'habitation pendant plus d'une année) qualifient ce phénomène de "voyages de travail quotidien" (ežednevni trudovi pătuvanija). D'autres, qui mettent l'accent dans leurs travaux sur les ressemblances dans le type de mobilité, ses origines et les réseaux au sein desquels elle intervient, ainsi que sur les effets des migrations tant sur les migrants que sur la région, préfèrent employer le terme de "migration de travail quotidienne". 
28. Voir Creed (Gerald), Domesticating Revolution : From Socialist Reform to Ambivalent Transition in a Bulgarian Village, University Park: The Pennsylvania State University Press, 1998, Pp. 168-169. Les chercheurs travaillant sur les migrations quotidiennes de travail sous le socialisme saluent leur développement dans la mesure où elles sont tenues pour une alternative à une migration définitive et au dépeuplement des villages, d'une part, et comme un instrument devant servir la modernisation de ces derniers, d'autre part. Voir Ninov (Z.), «Ežednevnite trudovi pătuvanija i razvitieto na urbanizacionnija proces» [Les migrations de travail quotidiennens et le développement du processus d'urbanisation], Izvestija na Bălgarskoto geografsko družestvo [Bulletin de l'Association de géographie bulgare], 28 (18), 1980 ; Nikolova (Marija), Ženite ot migriralite $v$ grada semejstva [Les femmes des familles ayant migré vers la ville], Sofia : AI "Prof. M. Drinov", 2003.

29. Voir Kalčev (Jordan), « Vătrešna i vănšna migracija na naselenieto » [Migration interne et internationale de la population], communication présentée lors du séminaire La stratégie européenne pour l'emploi et la Bulgarie - amélioration de l'adaptivité et de la mobilité de la main d'œuvre, 17-18 novembre 2005, Sofia, à l'adresse : http://www.eesbg.info/bg/activity/7-Sofia.htm.

30. Voir NSI, Prebrojavane na naselenieto..., (op.cit.), p.18.

31. Voir Sardon (Jean-Paul), "Demographic Change in the Balkans since the End of the 1980s ", Population: An English Selection, 13 (2), 2001, p. 56.

32. Voir NSI, Nătrešna $i$ vănšna migracija na naselenieto $v$ Bălgarija (rezultati ot representativno izučavane) [Migration interne et internationale de la population en Bulgarie (résultats d'une enquête représentative], à l'adresse : http://www.nsi.bg/Census/Vivmigr.htm. Viennent ensuite, dans la liste des motivations, la recherche des conditions de vie meilleures (22\%), d'un avenir meilleur pour les enfants (15\%), de l'éducation souhaitée (1\%), de revenus plus élevés (8\%), etc.

33. Voir Rapport annuel du ministère de la Jeunesse et des Sports, 2004, p.7.

34. NSI, Naselenie i demografski procesi prez 2005 [La population et les processus démographiques en 2005] (Sofia, 2006).

35. La restitution des terres agricoles à leurs anciens propriétaires et à leurs descendants a débuté en 1991. La Loi sur la propriété et sur l'utilisation des terres agricoles alors adoptée a subi des amendements en 1992 et en 1995, liés aux changements de majorité politique (gouvernement des ex-communistes, de l'opposition démocratique puis, de nouveau, des ex-communistes). Ces initiatives juridiques ont eu pour effet une liquidation des coopératives socialistes et une fragmentation extrême de la propriété agricole. Le principe retenu était celui d'une restitution des terres dans leurs frontières réelles de 1946- une année qui avait marqué l'apogée du morcellement des terres dans l'histoire moderne de la Bulgarie, puisque $92 \%$ des terres agricoles y occupaient alors une superficie inférieure à 10 hectareset seulement $1 \%$ dépassaient les 20 hectares.54\% de la population a déposé une demande de restitution ; des requêtes ont également été soumises par des églises, des mosquées, des communes, l'État et d'autres institutions.Comme moins de 13\% des terres n'avaient subi aucun changement de découpage depuis 1946, une redistribution des terres s'est imposée qui a rendu le processus de restitution exceptionnellement âpre, entravé par une multitude d'objections, de plaintes formelles et de disputes enflammées entre nouveaux propriétaires, comme entre ces derniers et l'État. En 2004, la taille moyenne des parcelles agricoles était de 0,6 hectares.Voir Giordano (Christian), Kostova (Dobrinka), "The Social Production of Mistrust ", in Hann (Chris), ed., Postsocialism. Ideals, Ideologies and Practices in Eurasia Londres : Routledge, 2002 ; Creed (Gerald), op.cit. ; Strong (Ann Louise), Restitution of Farm Land in Bulgaria, 1995, à l'adresse : http://www.ssc.upenn.edu/ east/spring95/strong.html. Voir aussi Iliev (Ilia), "The Division of Labor between Small and Large Farms in Bulgaria: A Four Decade Anomaly ", in Kabakchieva (Petja), Avramov (Rumen), eds., East-West Cultural Encounters. Entrepreneurship, Governance, Economic Knowledge, Sofia: n.d., 2004. 
36. Le financement pluriannuel de pré-adhésion au titre du programme SAPARD de la Commission européenne a été adopté en 2000. Il prévoyait que 592961125 euros (un chiffre comprenant les financements communautaires et la co-financement bulgare) soient accordés aux régions rurales d'ici l'adhésion de la Bulgarie à l'UE en 2007. Depuis l'intégration européenne de 2007, un Programme pour le développement des régions rurales (2007-2013) est en vigueur, en vertu duquel 4278384487 Euros (un chiffre qui comprend le financement communautaire, le cofinancement national et des contributions privées) devaient être octroyés à la Bulgarie à travers le Fonds européen de développement régional (FEDER). Voir Programa za razvitie na selskite rajoni (2007-2013) [Programme pour le développement des régions rurales], décembre 2007, p.82, p.234, à l'adresse :http://www.mzh.government.bg/Articles/363/Files/

RDP_official_version_BG\%20_2633468019477500000.pdf.

37. La réforme du système de retraites introduite en 1998-2001 est considérée, y compris par les experts en matière d'assurance sociale, comme "exceptionnellement restrictive" du point de vue du niveau des retraites proposé (en pourcentage du revenu) et des conditions d'éligibilité. Le rapport entre le montant moyen des retraites et le salaire bulgare moyen était, en 2001, de $34 \%$ (43\% en 2007). Dans le même temps, les retraites ont été plafonnées : jusqu'en 2004, elles ne pouvaient excéder quatre fois le montant de la pension sociale. Depuis 2004, elles sont limitées à $35 \%$ du revenu d'assurances maximum (soit 1000 euros en 2008). L'âge de départ à la retraite a été porté de 60 à 63 ans pour les hommes et de 55 à 60 ans pour les femmes. La durée de cotisation donnant droit à une retraite - qui était, dans le régime précédent, de 25 ans pour les hommes et de 20 ans pour les femmes (à l'exclusion de quelques groupes bénéficiant de régimes spéciaux) - a été augmentée de fait par l'introduction d'un système de points (100 pour les hommes, 94 pour les femmes).Cette réforme s'explique par l'incapacité financière de l'État à couvrir les droits de retraite accumulés dans le cadre du précédent système - plus généreux - et par le désir d'éviter un effondrement financier du système de retraite. Voir Tafradjiyski (Borislav), Lukanova (Pobeda), Shopov (George), Staykova (Dochka), The Pension Reform in Bulgaria - Two Years After the Start, 2001: http://www.ier.hit-u.ac.jp/pie/Japanese/ discussionpaper/dp2001/dp64/text.pdf; Hristoskov (Jordan), Bulgarian Pension Reform, presentation at the Moldova Pension Reform Workshop, Chisinau, June 10-11, 2008, à l'adresse: http://siteresources.worldbank.org/INTMOLDOVA/Resources/

Bulgarian_Pension_System_en.ppt.

38. Voir Sardon (Jean-Paul), art.cit., p.55.

39. Ibid.

40. Voir Fassmann (Heinz), Munz (Rainer), «European East-West Migration, 1945-1992 », International Migration Review, 28 (3), 1994, p.531.

41. Voir NSI, Prebrojavane na naselenieto..., (op.cit.), pp.30-34 ; Kalčev (Ivan), art.cit.

42. Six régions de planification ont alors été créées en vue de l'intégration de la Bulgarie à l'UE et de la gestion du développement régional avec l'aide des Fonds structurels communautaires. Ces régions ne correspondent pas à des unités territoriales ayant connu une existence antérieure; elles ne reflètent pas non plus des liens sociaux ou économiques d'ores et déjà existants. Dans la mesure où il ne s'agit pas d'entités administratives-territoriales, elles ne sont utilisées qu'à des fins de statistique régionale et de planification. En 2006, les frontières de presque toutes les régions ont été modifiées pour répondre aux exigences d'Eurostat (une région ne doit pas comprendre moins de 800000 habitants). Voir http://www.europe.bg/htmls/ page.php?id=7707\&category=82.

43. Voir NSI, Statističeski godišnik [Annuaire statistique], Sofia : 2004, qui contient des données de 2003 ; NSI, Statističeski godišnik [Annuaire statistique], Sofia: 2003 (données de 2002); NSI, Statističeski godišnik [Annuaire statistique], Sofia : 2002 (données de 2001).

44. Voir NSI, Prebrojavane na naselenieto..., (op. cit.), p. 21. Selon le dernier recensement de 2001, les Bulgares représentent $83,9 \%$ de la population, suivis par les Turcs $(9,4 \%)$ et les Roms $(4,7 \%)$. 
Voir http://www.nsi.bg/Census/Census.htm.Concernant les migrations roms, voir aussi Pamporov (Aleksej), «Demografskite harakteristika na romskite migracij v Bălgarija » [Les caractéristiques démographiques des migrations roms en Bulgarie], Naselenie, 13 (1-2), 2002.

45. Voir Nacionalnata programa za podobrjavane na žilištnite uslovija na romite v Republika Bălgarija za perioda 2005-2015 [Programme national pour l'amélioration des conditions de logement des Roms en République de Bulgarie sur la période 2005-2015],http://ethnos.bg/index.php? $\mathrm{TPL}=2 \& \mathrm{MID}=423 \& \mathrm{SID}=423$.

46. Voir Denkov (Dimităr), Stanoeva (Elica), Vidinski (Vasil), Romskite učilišta v Bălgarija [Les écoles roms en Bulgarie], Sofia : Fondacija Otvoreno Obštestvo, 2001, à l'adresse : http:// www.osi.hu/esp/rei/romaschools.bg.osf/bg/the_problem.html; voir aussi Mihajlov (Dočo), Željazkova (Antonina), Okončatelen doklad po proekt : ocenka za săštestvuvaštite obrazovatelni politiki i praktiki za predostavjane na raven dostăp do obučenie na deca ot malcinstvata i za razrabotvane na preporăki za ustojčivo rešenie za obrazovatelnite problemi na malcinstvata [Rapport final du projet : évaluation des politiques et des pratiques éducatives existantes concernant l'égal accès des enfants des minorités à l'enseignement et élaboration de recommandations pour une résolution efficace des problèmes des minorités], Sofia : IMIR, 2003, à l'adresse : http://www.imir-bg.org/ imir/reports/obrazovanie-malcinstvata.pdf. Les auteurs de l'études affirment qu'il y a en Bulgarie en 2002-2003 des écoles dans lesquelles entre 50 et $100 \%$ des élèves sont roms, desservant des villages qui sont principalement ou entièrement roms, ainsi que des quartiers et des ghettos urbains. Toutefois, d'après eux, cette ségrégation à l'école n'est pas imputable à un phénomène de discrimination, mais aux migrations intensives de l'après-1989, différenciées selon les groupes ethniques. D'après A. Željazkova et D. Mihajlov, la concentration des Roms dans certains villages, quartiers et ghettos ne doit pas seulement aux tendances démographiques enregistrées dans le pays, mais également à un repli ethnocommunautaire (etnokapsulacija) provoqué par l'appauvrissement radical et par un désir croissant d'organisation spatiale séparée des Roms à des fins de survie. Le Comité Helsinki bulgare (BHK) a également mis l'accent sur la nécessité d'œuvrer à une déségrégation des écoles devenues de facto roms sous l'effet des migrations ou d'autres facteurs dans certains villages restés jusqu'à ce jour en dehors du champ de l'action publique. Voir Po pătja kăm zrelostta. Ocenka na desegregacionnija proces, osăštestjavan ot nepravitelstveni organizacii $v$ Bălgarija [Sur la voie de la maturité. Evaluation du processus de déségrégation mis en œuvre par des organisations non gouvernementales en Bulgarie], Sofia : BHK, 2008, pp.16-17, à l'adresse : http://www.bghelsinki.org/upload/resources/ Po_patya_kam_zrelostta.pdf.

47. Voir http://ef.mlsp.government.bg/bg/page_op.php?id=1433[consultée le 3 septembre 2008].

48. Dans deux Rapports successifs sur les avancées de la Bulgarie en matière de satisfaction des exigences du Rapport commun portant sur l'évaluation des priorités de la politique de l'emploi en Bulgarie (2004 et 2005) du Ministère du Travail et des Affaires sociales, sont répertoriées les initiatives prises en matière de renforcement de la mobilité de travail. Ainsi, en 2003, afin d'accroître la mobilité territoriale des chômeurs ayant été embauchés ailleurs que sur le lieu de travail (art. 42 de la Loi sur la stimulation de l'activité de décembre 2001 prévoyant le versement d'une aide au déménagement des meubles ; le remboursement des dépenses quotidiennes liées au transport depuis et vers le lieu de travail ; remboursement des frais de transport relatifs à la présentation devant l'employeur) ont été alloués 33798 leva (approximativement la moitié en euros) pour 362 chômeurs; en 2004, à la même fin ont été dépensés 79015 leva pour 642 chômeurs. Les documents cités dans le paragraphe précédent comme dans celui-ci peuvent être consultés sur le site du ministère de Travail et des Affaires sociales à l'adresse: http:// www.mlsp.government.bg/bg/docs/index.htm [consultée le 3 septembre 2008].

49. Voir Wimmer (Andreas), Glick-Schiller (Nina), "Methodological Nationalism and the Study of Migration », Archives européennes de sociologie, 93 (2), 2002, p.217. 
50. Deneva (Neda), «The Role of Ethnicity in the Re-construction of the Community: Internal and International Migration in a Bulgarian Muslim Village », in Szczepaniková (Alice), Čaněk (Marek), Grill (Jan), eds., Migration Processes in Central and Eastern Europe: Unpacking the Diversity (Multicultural Center Prague: Prague, 2006), http://aa.ecn.cz/img_upload/ 79a33131c9c4293e0fcefb50bfa263ef/Migration_Processes_in_CEE_MKC.pdf.

51. Ce paragraphe, ainsi que les deux suivants, reprennent des points développés dans Guentcheva (Rossitza), Kabakchieva (Petya), Kolarski (Plamen), Migration Trends in Selected EU Applicant Countries. Vol. I - Bulgaria : The Impact of Seasonal Migration, IOM : Vienna, 2004, pp.50-51.

52. Voir, par exemple, K. Bruce Newbold, «Internal Migration of the Foreign-Born in Canada ", International Migration Review, 30( 3), 1996, p.728-747; Douglas Gurak and Mary Kritz, "The Interstate Migration of U.S. Immigrants : Individual and Contextual Determinants ", Social Forces, 78 (3), 2000, pp.1017-1039.

53. De concert avec la migration des étudiants et celle de personnes à haute qualification. Voir Ettore Recchi, Damian Tambini, Emiliana Baldoni, David Williams, Kristin Surak and Adrian Favell, « Intra-EU Migration : A Socio-Demographic Overview », PIONEUR Working Paper, 3, juillet 2003, à l'adresse : http://www.obets.ua.es/pioneur/documentos_public.php [consultée le 25 août 2008].

\section{RÉSUMÉS}

L'article analyse les tendances des migrations internes en Bulgarie au cours des quinze années qui ont suivi la chute du régime socialiste en les confrontant aux processus migratoires antérieurs à 1989. Il attire l'attention sur les flux migratoires entre ville et village, sur le profil des migrants, sur leur occupation économique et sur les migrations de travail quotidiennes. Ce faisant, il souligne l'existence de continuités intéressantes entre l'avant et l'après-1989 dans les aspirations de l'Etat bulgare à contrôler et à orienter les migrations internes. Les instruments mis au service de cette politique ont pu changer, tout comme les priorités définies, mais l'ambition de promouvoir une politique de peuplement qui soit placée au service d'autres finalités étatiques, notamment économiques, est demeurée. Enfin, l'article suggère quelques pistes pour penser les migrations internes dans leurs relations avec les migrations externes, mettant en exergue les thèmes, les problèmes et le champ que de telles recherches pourraient souhaiter explorer.

\section{INDEX}

Index géographique : Bulgarie

Mots-clés: Mobilités, Migrations internes

\section{AUTEURS}

\section{ROSSITZA GUENTCHEVA}

Dr. Rossitza Guentcheva est maître de conférence (asistent) au Département d'anthropologie de la Nouvelle université bulgare à Sofia. Elle est titulaire d'une maîtrise en histoire de l'Université 
de Sofia « Kliment Ohridski » (1992), d'un Master en histoire de la Central European University à Budapest (1995) et d'un doctorat en histoire de l'Université de Cambridge (2001). Ses recherches portent sur les migrations et la mobilité, l'anthropologie de l'Europe de l'Est, l'histoire sociale et culturelle du socialisme et l'histoire sociale de la langue. Elle a récemment publié : « Images of the West in Bulgarian Travel Writing during Socialism (1945-1989) », in : Wendy Bracewell and Alex Drace-Francis (eds), Under Eastern Eyes: A Comparative Introduction to East European Travel Writing on Europe, Budapest \& New York : Central European University Press, 2008, p.355-378; «From Banishment to Ascribed Residence : Controlling Internal Movement in Socialist Bulgaria (1944-1989) », paper in the framework of the project « Roles, Identities and Hybrids » of the Centre for Advanced Study - Sofia, May 2006; «Sounds and Noise in Socialist Bulgaria », in : John Lampe and Mark Mazower (eds.), Ideologies and National Identities. The Case of Twentieth-Century Southeastern Europe, Budapest : Central European University Press, 2004, p.211-234. mailto:mrossitza.guentcheva@gmail.com 